\title{
A complex Ruelle-Perron-Frobenius theorem and two counterexamples
}

\author{
MARK POLLICOTT \\ Mathematics Institute, University of Warwick, Coventry, CV4 7AL, England
}

(Received 8 July 1983)

\begin{abstract}
In this paper a new proof of a theorem of Ruelle about real PerronFrobenius type operators is given. This theorem is then extended to complex Perron-Frobenius type operators in analogy with Wielandt's theorem for matrices. Finally two questions raised by Ruelle and Bowen concerning analyticity properties of zeta functions for flows are answered.
\end{abstract}

\section{Introduction}

The operator $\mathscr{L}_{f}$ we shall be studying has its origins in statistical mechanics. In this context it is only necessary to consider its action on the space of real-valued functions (or interactions) of exponentially decreasing variation $\mathscr{F}_{\theta}$. Ruelle showed that the spectrum of $\mathscr{L}_{f}: \mathscr{F}_{\theta} \rightarrow \mathscr{F}_{\theta}\left(f \in \mathscr{F}_{\theta}\right)$ satisfies a Perron-Frobenius type theorem (theorem 1) [15]. Subsequently other proofs of this, and other related results, have been developed [20], [4], ([17, p. 83]). In $\S 1$, we present a new proof of the existence of a maximal eigenvalue for $\mathscr{L}_{f}$.

One major application of Ruelle's theorem is the construction of meromorphic extensions for certain generalized zeta functions [16].

It is the purpose of this paper to present a generalization of this theorem to describe the spectrum of $\mathscr{L}_{f}$ for complex functions of exponentially decreasing variation (theorem 2). This subsumes a complex version of the Perron-Frobenius theorem for matrices due to Wielandt (proposition 1). This new spectral theorem provides a more natural setting for the ingenious techniques developed by Ruelle ([17, pp. 93-95]), and enables us to produce extension results for zeta functions (theorem 3) subsuming those due to Ruelle [16] and Parry and the author ([12, theorem 1]).

In the last two sections we give examples which answer negatively questions raised by Ruelle $([17$, p. 173$])$ and Bowen $([1$, p. 31$])$. (During the writing of this paper the author discovered that the example in $\S 4$ was known to Ruelle [18].)

This paper is an offshoot of the joint work of Parry and the author concerning asymptotic estimates for the number of closed orbits for Axiom A flows [12]. Parry has since derived other interesting estimates as a result of applying ideas from analytic number theory to the study of Axiom A flows [11]. 
I wish to express my gratitude to the S.E.R.C. for their financial support. I am deeply indebted to Professor William Parry for his help and encouragement throughout the course of this work.

1. The Ruelle operator theorem

Let $\mathrm{A}$ be an aperiodic zero-one matrix of rank $k$ and define

$$
\Sigma_{A}^{+}=\left\{x \in \prod_{0}^{\infty}\{1,2, \ldots, k\} \mid A\left(x_{n}, x_{n+1}\right)=1\right\} .
$$

The space $\Sigma_{A}^{+}$is compact and zero dimensional with respect to the topology with basis consisting of sets of the form $\left\{x \in \Sigma_{A}^{+} \mid x_{i}=z_{i}, 0 \leq i \leq n-1\right\}$.

The (one-sided) shift of finite type $\sigma: \Sigma_{A}^{+} \rightarrow \Sigma_{A}^{+}$is the continuous map given by $(\sigma x)_{n}=x_{n+1}$. Since $A$ is aperiodic $\sigma$ is (topologically) mixing.

If $f: \Sigma_{A}^{+} \rightarrow \mathbb{R}$ is continuous, the pressure is defined by

$$
P(f)=\sup \left\{h_{\mu}(\sigma)+\int f d \mu \mid \mu \text { is } \sigma \text {-invariant }\right\} .
$$

This supremum is always attained and the measures for which $P(f)=h_{\mu}(\sigma)+\int f d \mu$ are called equilibrium states ([21, p. 224]).

Define $\operatorname{var}_{n} f=\sup \left\{|f(x)-f(y)| \mid x_{i}=y_{i}, 0 \leq i \leq n-1\right\}$ then for $0<\theta<1$ let

$$
\|f\|_{\theta}=\sup \left\{\frac{\operatorname{var}_{n} f}{\theta^{n}} \mid n \geq 0\right\} \text {. }
$$

In this section our main interest will be in the real Banach space

$$
\mathscr{F}_{\theta}=\left\{f \in C\left(\Sigma_{A}^{+}\right) \mid\|f\|_{\theta}<\infty\right\}
$$

with norm $\|f\|_{\theta}=\max \left\{\|f\|_{\infty},\|f\|_{\theta}\right\}$. Given $f \in \mathscr{F}_{\theta}$ define an operator $\mathscr{L}_{f}: \mathscr{F}_{\theta} \rightarrow \mathscr{F}_{\theta}$ by

$$
\mathscr{L}_{f} h(z)=\sum_{\sigma y=z} \exp (f(y)) h(y) .
$$

We now present a proof of the Ruelle operator theorem which does not involve measures. The existence part was suggested by techniques employed by Krasnoselskii in [9] for a different problem. The rest of the proof is a combination of Ruelle ([17, p. 90]) and Walters [20].

THEOREM 1 (Ruelle). Let $\sigma$ be a topologically mixing one-sided shift of finite type and let $f \in \mathscr{F}_{{ }}$. The operator $\mathscr{L}_{f}$ has $e^{P(f)}$ as a simple eigenvalue (with a positive eigenvector). Furthermore the rest of the spectrum is contained in a disc of radius strictly smaller than $e^{P(f)}$.

Proof. Let $\Lambda$ denote the \|\|$_{\infty}$-closed set of positive continuous functions $g: \Sigma_{A}^{+} \rightarrow \mathbb{R}^{+}$ with $\|g\|_{\infty} \leq 1$ and

$$
g(x) \leq g(y) \exp \left(\theta^{n}\|f\|_{\theta} / 1-\theta\right)
$$

whenever $x_{i}=y_{i}, 0 \leq i \leq n-1$. Since

$$
|g(x)-g(y)| \leq\left(\theta^{n} /(1-\theta)\right)\|f\|_{\theta} \exp \left(\|f\|_{\theta} /(1-\theta)\right)
$$


for such $x$ and $y, \Lambda$ is equicontinuous and therefore \|\|$_{\infty}$-compact by the ArzelaAscoli theorem.

The continuous map $L_{n}: \Lambda \rightarrow \Lambda$ given by

$$
L_{n} g=\mathscr{L}_{f}(g+1 / n) /\left\|\mathscr{L}_{f}(g+1 / n)\right\|_{\infty} \quad n \geq 0
$$

has a fixed point $h_{n}$ by the Schauder-Tychonoff theorem. If $\lambda_{n}$ denotes $\left\|\mathscr{L}_{f}\left(h_{n}+1 / n\right)\right\|_{\infty}$ then

$$
\begin{aligned}
\lambda_{n} h_{n}=\mathscr{L}_{f}\left(h_{n}+1 / n\right) & \geq\left(\inf h_{n}+1 / n\right) e^{-\|f\|_{\infty}} \\
& >0
\end{aligned}
$$

and so $\lambda_{n}>e^{-\|f\|_{\infty}}$. If $h$ is a limit point of $\left\{h_{n}\right\}$ then $\mathscr{L}_{f} h=\lambda h$, where $\lambda=\left\|\mathscr{L}_{f} h\right\|_{\infty}>0$. The eigenfunction $h$ is strictly positive since if $h(x)=0$ for some $x$ then

$$
\mathscr{L}_{f}^{n} h(x)=\sum_{\sigma^{n} y=x} \exp \left(f^{n}(y)\right) h(y)=0,
$$

(where $f^{n}(y)=f(y)+f(\sigma y)+\cdots+f\left(\sigma^{n-1} y\right)$ ). This would make $h$ zero on the dense set $\left\{y \mid \sigma^{n} y=x\right.$ for some $\left.n \geq 0\right\}$ contradicting $\lambda>0$.

The eigenvalue $\lambda$ is simple since if $\mathscr{L}_{f} g=\lambda g$ and $t=\inf \{g / f\}$ then $(t f-g)(x)=0$ for some $x$. The preceding argument applied to $t f-g \geq 0$ shows that $t f=g$.

By replacing $f$ by $f+\log h-\log h \circ \sigma-\log \lambda$ we may assume that $\mathscr{L}_{f} 1=1$. The general effect of this change is to scale the spectrum of $\mathscr{L}_{f}$ by $\lambda$.

Since $A$ is aperiodic, $A^{M}>0$ for some $M$. Given $z, x$ choose $y \in\left\{\sigma^{-n-M} z\right\}$ with $x_{i}=y_{i}, 0 \leq i \leq n-1$. Then

$$
f^{n}(x) \leq f^{n+M}(y)+M\|f\|_{\infty}+\left(\|f\|_{\theta} /(1-\theta)\right)
$$

and

$$
\sum_{\sigma^{n} x=x} \exp \left(f^{n}(x)\right) \leq k \cdot \sum_{\sigma^{n+M} M_{y=z}} \exp \left(f^{n+M}(y)\right)=k \cdot 1 \quad(k>0)
$$

Thus $P(f)=\lim _{n \rightarrow \infty}(1 / n) \log \sum_{\sigma^{n} x=x} \exp \left(f^{n}(x)\right)=0$, by use of the variational principle ([21, p. 218]). Thus for our original $f, \log \lambda=P(f)$. It is easy to show that (for some constant $C>0$ )

$$
\left\|\mathscr{L}_{f}^{n} g\right\|_{\theta} \leq C\|g\|_{\infty}+\theta^{n}\|g\|_{\theta}
$$

for all $g \in \mathscr{F}_{\theta}, n \geq 0$. This means that $\left\{\mathscr{L}_{f}^{n} g\right\}$ is equicontinuous with respect to \|\|$_{\infty}$ and there exists a limit point $l$. If we write $\alpha(g)=\sup \{g(x)\}$ and $\beta(g)=\inf \{g(x)\}$ then

$$
\alpha(g) \geq \alpha\left(\mathscr{L}_{f} g\right) \geq \cdots \geq \alpha(l)=\alpha\left(\mathscr{L}_{f}^{n} l\right) \quad n \geq 0
$$

and

$$
\beta(g) \leq \beta\left(\mathscr{L}_{f} g\right) \leq \cdots \leq \beta(l)=\beta\left(\mathscr{L}_{f}^{n} l\right) \quad n \geq 0 .
$$

Since $\sigma$ is mixing the equalities show $l$ is a constant. Furthermore since $\alpha(l)=\beta(l)$ the sequence $\mathscr{L}_{f}^{n} g$ converges uniformly to $l$.

To remove the maximal eigenvalue consider $\mathscr{L}_{f}$ acting on the quotient space $\mathscr{F}_{\theta} / \mathbb{R}$. On this space $(1.1)$ becomes

$$
\left\|\mathscr{L}_{f}^{n} g\right\|_{\theta} \leq \mathrm{C} \cdot \operatorname{var}_{0} \mathrm{~g}+\theta^{n}\|g\|_{\theta} .
$$

Since $\operatorname{var}_{0} \mathscr{L}_{f}^{n} g$ converges to zero we have for large $n$

$$
\left\|\mathscr{L}_{f}^{2 n} g\right\|_{\theta} \leq C \cdot \operatorname{var}_{0} \mathscr{L}_{f}^{n} g+\theta^{n}\left[C \cdot \operatorname{var}_{0} g+\theta^{n}\|g\|_{\theta}\right]
$$


By the uniform compactness of $\left\{g \mid\|g\|_{\theta} \leq 1\right\}$ we may choose $n$ so that (1.2) holds for all $g$ in this ball. Thus the spectral radius of $\mathscr{L}_{f}: \mathscr{F}_{\theta} / \mathbb{R} \rightarrow \mathscr{F}_{\theta} / \mathbb{R}$, denoted by $\rho\left(\mathscr{L}_{f}\right)$, satisfies

$$
\rho\left(\mathscr{L}_{f}\right)=\inf \left\{\left\|\mathscr{L}_{f}^{n}\right\|_{\theta}^{1 / n} \mid n \geq 0\right\}<1 .
$$

This completes the proof.

\section{A complex Ruelle operator theorem}

A continuous function $f: \Sigma_{A}^{+} \rightarrow \mathbb{C}$ is called locally constant if there exists $n>0$ such that $f(x)$ depends on only the first $n$ places of $x$, i.e. $f(x)=f(y)$ whenever $x_{i}=y_{i}$, $0 \leq i \leq n-1$.

The operator $\mathscr{L}_{f}$ leaves invariant the finite dimensional subspace of $C\left(\Sigma_{A}^{+}, \mathbb{C}\right)$ with base vectors

$$
\delta_{x_{0}, \ldots, x_{n-2}}(x)= \begin{cases}1 & x_{i}=z_{i}, 0 \leq i \leq n-2 \\ 0 & \text { otherwise }\end{cases}
$$

It is always possible to reduce locally constant functions to the case $n=2$ by considering $x \in \Sigma_{A}^{+}$as the sequence $\left(x_{m}, \ldots, x_{m+n-2}\right)_{m=0}^{\infty}$ in a shift space whose symbols are words of length $n-1$.

For $n=2, \mathscr{L}_{f}$ can be represented by a matrix

$$
M=\left[A\left(x_{0}, x_{1}\right) \cdot \exp f\left(x_{0}, x_{1}\right)\right] .
$$

When $f$ is real-valued the eigenvalues of $M$ are described by the Perron-Frobenius theorem. More generally define $M_{+}$to be the positive matrix with entries $\left|M\left(x_{0}, x_{1}\right)\right|$. The following result is due to Wielandt $([6, \mathrm{p} .57])$.

Proposition 1. The eigenvalues of $M$ have moduli less than or equal to the maximal eigenvalue $\beta$ for $M_{+}$. If $\beta e^{i a}$ is an eigenvalue of $M$ (for some $0 \leq a<2 \pi$ ) then $M$ takes the form $M=e^{i a} D M_{+} D^{-1}$ where $D$ is a diagonal matrix with diagonal entries of unit modulus.

Ruelle's theorem (theorem 1) can be viewed as a generalization of the PerronFrobenius theorem. In this section we present an analogous extension of Wielandt's result.

The space $\mathscr{F}_{\theta}^{\mathbb{C}}=\left\{f \in C\left(\Sigma_{A}^{+}, \mathbb{C}\right) \mid\|f\|_{\theta}<\infty\right\}$ is a complex Banach space with norm $\|f\|_{\theta}=\max \left\{\|f\|_{\theta},\|f\|_{\infty}\right\}$. (Here $\|\cdot\|_{\theta}$ has the same definition as in $\S 1$.) If $f=u+i v \epsilon$ $\mathscr{F}_{\theta}^{\mathbb{C}}$ then $u, v \in \mathscr{F}_{\theta}$ and we freely assume that $\mathscr{L}_{u} 1=1$, as in the proof of theorem 1 .

For $g \in C\left(\Sigma_{A}^{+}\right)$let $\Gamma_{g}$ be the multiplicative group generated by $\left\langle\exp g^{n}(x) \mid \sigma^{n} x=x\right\rangle$ (where $g^{n}(x)=g(x)+g(\sigma x)+\cdots+g\left(\sigma^{n-1} x\right)$ ), [13], [12].

Proposition 2. For $f=u+i v \in \mathscr{F}_{\theta}^{\mathbb{C}}$ and $0 \leq a<2 \pi$ the following are equivalent:

(i) $\Gamma_{v-a}$ is generated by a power of $e^{2 \pi}$;

(ii) $\lambda_{a} \equiv e^{[i a+P(u)]}$ is an eigenvalue for $\mathscr{L}_{f}$;

(iii) there exists $\omega \in C\left(\Sigma_{A}^{+}\right)$such that

$$
v-a+\omega \circ \sigma-\omega \in C\left(\Sigma_{A}^{+}, 2 \pi \mathbb{Z}\right) .
$$


Proof. (i) $\Rightarrow$ (ii) Choose $x \in \Sigma_{A}^{+}$with a dense orbit and define $h$ on $\left\{\sigma^{n} x \mid n \geq 0\right\}$ by $h\left(\sigma^{n} x\right)=\exp i(v-a)^{n}(x)$. This extends to an element of $\mathscr{F}_{\theta}^{\mathrm{C}},[10]$. Furthermore $h$ satisfies $h(\sigma z)=h(z) \exp i(v-a)(z)$ and consequently $\mathscr{L}_{f} h=e^{i a} h$.

(ii) $\Rightarrow$ (iii) Since $\mathscr{L}_{f} h=e^{i a} h$ may be expressed as

$$
\sum_{\sigma y=x} \exp u(y) \exp i(v-a)(y) h(y)=h(x)
$$

if $|h(x)|$ is maximal then so is $|h(y)|$ when $\sigma y=x$. Because $\sigma$ is mixing $h$ is of constant modulus. Thus (2.1) represents a convex combination of points on a circle which also lies on the circle. From this we deduce

$$
\exp i(v-a)(y) h(y)=h(\sigma y),
$$

or equivalently $v-a+\arg h-\arg h \circ \sigma \in C\left(\Sigma_{A}^{+}, 2 \pi \mathbb{Z}\right)$.

(iii) $\Rightarrow$ (i) This is immediate.

If $f \in \mathscr{F}_{\theta}^{\mathrm{C}}$ satisfies one, and hence all, of the above conditions we call it an a-function. For example, the functions in $\mathscr{F}_{\theta}$ are all $a$-functions with $a=0$. If $f$ is not an $a$-function (for any $a$ ) then we call it regular.

If $f=u+i v$ where $v-a+\omega \circ \sigma-\omega \in C\left(\Sigma_{A}^{+}, 2 \pi \mathbb{Z}\right)$ then

$$
\mathscr{L}_{f}=e^{i a} \Delta\left(e^{-i \omega}\right) \mathscr{L}_{u} \Delta\left(e^{i \omega}\right)
$$

where $\Delta(h)$ denotes the operator that multiplies functions by $h$. Therefore the spectrum of $\mathscr{L}_{f}$ is precisely the spectrum of $\mathscr{L}_{u}$ rotated through an angle $a$. By theorem 1:

Proposition 3. If $f=u+i v$ is an a-function then $\lambda_{a}$ is a simple eigenvalue for $\mathscr{L}_{f}$ and the rest of the spectrum is contained in a disc of radius strictly smaller than $\left|\lambda_{a}\right|=e^{P(u)}$.

An immediate corollary is that $f \in \mathscr{F}_{\theta}^{\mathrm{C}}$ can be an $a$-function for at most one $a$ $(0 \leq a<2 \pi)$.

For any $f=u+i v$ (with $\mathscr{L}_{u} 1=1$ ) the following extension of (1.1) is true:

$$
\left\|\mathscr{L}_{f}^{n} h\right\|_{\theta} \leq C \cdot\|h\|_{\infty}+\theta^{n}\|h\|_{\theta} \quad n \geq 0, h \in \mathscr{F}_{\theta}^{\mathbb{C}} .
$$

The operator norm satisfies $\left\|\mathscr{L}_{f}^{n}\right\|_{\theta} \leq C+1$ and we have an upper bound on the spectral radius

$$
\rho\left(\mathscr{L}_{f}\right)=\inf \left\{\left\|\mathscr{L}_{f}^{n}\right\|_{\theta}^{1 / n} \mid n \geq 1\right\} \leq 1 .
$$

We now show that when $f$ is regular the spectrum of $\mathscr{L}_{f}\left(\operatorname{denoted} \mathrm{sp}\left(\mathscr{L}_{f}\right)\right.$ ) is disjoint from the unit circle.

Choose a point $e^{i t}$ on the circle, then for $\|h\|_{\theta} \leq 1$ write

$$
h_{N}=\frac{1}{N} \sum_{n=0}^{N-1} \mathscr{L}_{f-i r}^{n} h \quad(N \geq 1) .
$$

By (2.2) $h_{N}$ is contained in the uniformly compact set $\left\{g\left|\|\mid\| \|_{\theta} \leq C+1\right\}\right.$. When $f$ is regular $\left\|h_{N}\right\|_{\infty}$ must tend to zero since any non-zero limit point of $\left\{h_{N}\right\}$ would be an eigenvector for $\mathscr{L}_{f}$ with eigenvalue $e^{i t}$. For $k \geq 0$,

$$
\left\|h_{N}\right\|_{\theta} \leq\left\|h_{N}-\mathscr{L}_{f-i t}^{k} h_{N}\right\|_{\theta}+\left\|\mathscr{L}_{f-i t}^{k} h_{N}\right\|_{\theta}
$$


where we have estimates

$$
\begin{aligned}
\left\|h_{N}-\mathscr{L}_{f-i t}^{k} h_{N}\right\|_{\theta} & =\left\|\frac{1}{N} \sum_{n=0}^{k-1} \mathscr{L}_{f-i t}^{n} h-\mathscr{L}_{f-i t}^{n+N} h\right\|_{\theta} \\
& \leq(2 k / N)(C+1),
\end{aligned}
$$

and

$$
\begin{aligned}
\left\|\mathscr{L}_{f-i t}^{k} h_{N}\right\|_{\theta} & \leq C\left\|h_{N}\right\|_{\infty}+\theta^{k}\left\|h_{N}\right\|_{\theta} \\
& \leq C \cdot\left\|h_{N}\right\|_{\infty}+\theta^{k}(C+1) .
\end{aligned}
$$

If we take $k=\left[N^{\frac{1}{2}}\right]$ then these bounds show that $\varlimsup\left\|h_{N}\right\|_{\theta}=0$. By the uniform compactness of $\left\{h \mid\|h\|_{\theta} \leq 1\right\}$ we can choose $N$ such that $\left\|h_{N} \mid\right\|_{\theta}<1$ for all $h$ in this ball. Since the spectral radius of an operator is smaller than its norm

$$
\rho\left(\frac{1}{N} \sum_{n=0}^{N-1} \mathscr{L}_{f-i t}^{n}\right) \leq\|\| \frac{1}{N} \sum_{n=0}^{N-1} \mathscr{L}_{f^{-i t}}^{n}\|\|_{\theta}<1 .
$$

Thus 1 is not an element of $\operatorname{sp}\left((1 / N) \sum_{n=0}^{N-1} \mathscr{L}_{f-i t}^{n}\right)$. However we know from the spectral mapping theorem $([19$, p. 263]) that

$$
\operatorname{sp}\left(\frac{1}{N} \sum_{n=0}^{N-1} \mathscr{L}_{f-i t}^{n}\right)=\left\{\frac{1}{N} \sum_{n=0}^{N-1} \lambda^{n} \mid \lambda \in \operatorname{sp}\left(\mathscr{L}_{f-i t}\right)\right\} .
$$

Thus 1 cannot be an element of $\operatorname{sp}\left(\mathscr{L}_{f-i t}\right)=e^{-i t} \operatorname{sp}\left(\mathscr{L}_{f}\right)$, or equivalently $e^{i t}$ is not in the spectrum of $\mathscr{L}_{f}$ Since $e^{i t}$ was chosen arbitrarily and $\operatorname{sp}\left(\mathscr{L}_{f}\right)$ is closed we have the following.

THEOREM 2. Let $\sigma$ be a topologically mixing one-sided shift of finite type and let $f=u+i v \in \mathscr{F}_{\theta}^{\mathbb{C}}$.

(i) If $f$ is an a-function then $\lambda_{a}=e^{[i a+P(u)]}$ is a simple eigenvalue for $\mathscr{L}_{f}$ and the rest of the spectrum is contained in a disc of radius strictly smaller than $\left|\lambda_{a}\right|=e^{P(u)}$.

(ii) If $f$ is regular then the spectrum of $\mathscr{L}_{f}$ is contained in a disc of radius strictly smaller than $e^{P(u)}$.

It is possible to formulate a proof of part (ii) closer to the proof of theorem 1 by proceeding along the lines of propositions 13 and 14 in [12].

An important feature of the above theorem is that the type of spectrum for $\mathscr{L}_{f}$ is determined by $\mathscr{I}(f)$ and the size of the spectrum is given by $\mathscr{R}(f)$.

We can obtain a lower bound for the spectral radius $\rho\left(\mathscr{L}_{f}\right)$ in the regular case. If $f=u+i v$ then $\mathscr{L}_{u}^{n}=\mathscr{L}_{f}^{n} \Delta\left(e^{-i v^{n}}\right)$ and

$$
e^{P(u)}=\rho\left(\mathscr{L}_{u}\right) \leq \rho\left(\mathscr{L}_{f}\right) \cdot \varlimsup \lim \left\|\Delta\left(e^{-i v^{n}}\right)\right\|_{\theta}^{1 / n} .
$$

It is simple to show $\varlimsup\|m\|\left(e^{-i v^{n}}\right) \|_{\theta}^{1 / n} \leq 1 / \theta$ and so $\rho\left(\mathscr{L}_{f}\right) \geq \theta \cdot e^{P(u)}$.

Proposition 2(i) shows that a necessary condition for $u+i v$ to be an $a$-function (for some $a$ ) is that $\Gamma_{v}$ should be of rank at most two. But functions satisfying this condition can easily be approximated by functions which do not. This makes the family of regular functions dense in $\mathscr{F}_{\theta}^{\mathbb{C}}$. Furthermore, by theorem 2 and upper semicontinuity of $f \mapsto \rho\left(\mathscr{L}_{f}\right)$ this family is also open. 


\section{Extending the zeta function}

Given $f \in \mathscr{F}_{\theta}^{\mathbb{C}}$ define a zeta function by

$$
\zeta(f)=\exp \sum_{m=1}^{\infty} \frac{1}{m} \sum_{\sigma^{m} x=x} \exp f^{m}(x)
$$

where $f^{m}(x)=f(x)+f(\sigma x)+\cdots+f\left(\sigma^{m-1} x\right)$. If $f=u+i v$ with $P(u)<0$ then

$$
\begin{aligned}
& \varlimsup\left|\sum_{\sigma^{m} x=x} \exp f^{m}(x)\right|^{1 / m} \leq \varlimsup\left|\sum_{\sigma^{m_{x}=x}} \exp u^{m}(x)\right|^{1 / m} \\
& =e^{P(u)}<1 \text {. }
\end{aligned}
$$

Since convergence is uniform in a neighbourhood of $f$ it follows that $\zeta$ is non-zero and analytic on $\{f \mid \mathscr{R}(f)<0\}([17$, p. 100]). We now consider the cases where $P(u)=0$.

Proposition 4. If $f=u+i v \in \mathscr{F}_{\theta}^{\mathbb{C}}$ is regular with $P(u)=0$ then $\zeta$ is analytic and non-zero in a neighbourhood of $f$.

Proof. Choose $\theta^{\prime}>\theta$, then $f \in \mathscr{F}_{\theta}^{\mathbb{C}} \subseteq \mathscr{F}_{\theta^{\prime}}^{\mathbb{C}}$. For $n>0$ define locally constant functions

$$
\begin{array}{ll}
u_{n}(x)=\sup \left\{u(z) \mid x_{i}=z_{i},\right. & 0 \leq i \leq n-1\} \\
v_{n}(x)=\sup \left\{v(z) \mid x_{i}=z_{i},\right. & 0 \leq i \leq n-1\}
\end{array}
$$

and let $f_{n}(x)=u_{n}(x)+i v_{n}(x)$. This enables us to write

$$
\begin{aligned}
\zeta(f)= & \exp \sum_{m=1}^{\infty} \frac{1}{m} \sum_{\sigma^{m} x=x} \exp f^{m}(x)-\exp f_{n}^{m}(x) \\
& \times \exp \sum_{m=1}^{\infty} \frac{1}{m} \sum_{\sigma^{m} x=x} \exp f_{n}^{m}(x) .
\end{aligned}
$$

By applying theorem 2 to $\mathscr{L}_{f}: \mathscr{F}_{\theta^{\prime}}^{\mathbb{C}} \rightarrow \mathscr{F}_{\theta^{\prime}}^{\mathbb{C}}$, there exists $0<\beta<1$ such that spectral radius $\rho\left(\mathscr{L}_{f}\right)<\beta$. Since $g \mapsto \rho\left(\mathscr{L}_{g}\right)$ is upper semicontinuous on $\mathscr{F}_{\theta^{\prime}}^{\mathrm{C}}$ and

$$
\left\|f-f_{n}\right\|_{\theta^{\prime}} \leq\|f\|_{\theta}\left(\theta / \theta^{\prime}\right)^{n},
$$

it follows that $\rho\left(\mathscr{L}_{f_{n}}\right)<\beta$ for large enough $n$. If $\varepsilon$ is chosen sufficiently small then $\rho\left(\mathscr{L}_{g_{n}}\right)<\beta$ holds uniformly on $D=\left\{g\left|\|\mid g-f\|_{\theta}<\varepsilon\right\}\right.$.

Let $\lambda_{1}, \ldots, \lambda_{N(n)}$ be the eigenvalues of $\mathscr{L}_{f_{n}}$ acting on the finite dimensional invariant subspace of $\S 2$ then $\left|\lambda_{j}\right|<\beta, 1 \leq j \leq N(n)$. Furthermore

$$
\sum_{\sigma^{m} x=x} \exp f_{n}^{m}(x)=\operatorname{trace} \mathscr{L}_{f_{n}}^{m}=\lambda_{1}^{m}+\cdots+\lambda_{N(n)}^{m}
$$

where $N(n) \leq k^{n}$, ( $k$ is the dimension of $A$ ). Choose $\alpha$ satisfying $\beta k^{\alpha}<1$ and take $n=[m \alpha]$. Then

$$
\left|\sum_{\sigma^{m} x=x} \exp f_{n}^{m}(x)\right|^{1 / m} \leq\left(\beta^{m} k^{n}\right)^{1 / m} \leq \beta k^{\alpha}<1
$$

for sufficiently large $m$. Since this holds uniformly on $D$

$$
\sum_{m=1}^{\infty} \frac{1}{m} \sum_{\sigma^{m} x=x} \exp g_{n}^{m}(x)
$$

is analytic for $g \in D$. From the definitions of $u_{n}$ and $v_{n}$

$$
\begin{aligned}
& \left\|u_{n}^{m}-u^{m}\right\|_{\infty} \leq m\left\|u_{n}-u\right\|_{\infty} \leq m \theta^{n}\|u\|_{\theta} \\
& \left\|v_{n}^{m}-v^{m}\right\|_{\infty} \leq m \theta^{n}\|v\|_{\theta} .
\end{aligned}
$$


Since

$$
\begin{aligned}
\sum_{\sigma^{m} x=x} \exp f^{m}(x)-\exp f_{n}^{m}(x)= & \sum_{\sigma^{m} x=x}\left[\exp u^{m}(x)-\exp u_{n}^{m}(x)\right] \exp i v^{m}(x) \\
& +\sum_{\sigma^{m} x=x} \exp u_{n}^{m}(x)\left[\exp i v^{m}(x)-\exp i v_{n}^{m}(x)\right]
\end{aligned}
$$

it follows from (3.2) that

$$
\varlimsup\left|\sum_{\sigma^{m} x=x} \exp f^{m}(x)-\exp f_{n}^{m}(x)\right|^{1 / m} \leq \theta^{\alpha} e^{P(u)} .
$$

If we choose $\theta^{\alpha}<\Phi<1$ and $\varepsilon$ sufficiently small then for large $m$

$$
\left|\sum_{\sigma^{m} x=x} \exp g^{m}(x)-\exp g_{n}^{m}(x)\right|^{1 / m}<\Phi
$$

uniformly on $D$ and so

$$
\sum_{m=1}^{\infty} \frac{1}{m} \sum_{\sigma^{m} x=x} \exp g^{m}(x)-\exp g_{n}^{m}(x)
$$

is analytic on this disc. This completes the proof.

When $f$ is an $a$-function then $\rho\left(\mathscr{L}_{f}\right)=e^{P(\mathscr{R}(f))}$. However the isolated eigenvalue $\lambda_{a}$ can be dealt with using perturbation theory. In a neighbourhood of $f$ the operator $\mathscr{L}_{\mathrm{g}}$ still has an isolated eigenvalue $\beta$ ([3, p. 587]). This leads to a natural definition of the complex pressure (in a neighbourhood of an $a$-function) as $P(g)=\log \beta$.

By developing an approach due to Ruelle ([17, pp. 93-95]), Parry has proved the following result ([11, proposition 3$])$.

Proposition 5. If $f \in \mathscr{F}_{\theta}$ and $P(f)=0$ then there exists $\varepsilon>0$ such that $P$ extends to an analytic function in

$$
D=\left\{g||\|f-g\|_{\theta}<\varepsilon\right\}
$$

and

$$
\sum_{m=1}^{\infty}\left(e^{i a m} / m\right)\left(\sum_{\sigma^{m} x=x} \exp g^{m}(x)-e^{m P(g)}\right)
$$

converges uniformly in $D$.

Propositions 4 and 5 together give the following result; (the version for two-sided shifts is theorem 1 in [11]).

THEOREM 3. Let $f=u+i v \in \mathscr{F}_{\theta}^{\mathbb{C}}$.

(i) If $P(u)<0$ or $f$ is regular with $P(u)=0$ then $\zeta$ is non-zero and analytic in a neighbourhood of $f$.

(ii) If $f$ is an a-function with $P(u)=0$ then $\zeta$ has a non-zero analytic extension to a set $\left\{g\left|\|\mid g-f\|_{\theta}<\varepsilon, P(g) \neq 0\right\}\right.$ given by

$$
\zeta(g)=\frac{1}{1-e^{P(g)}} \exp \sum_{m=1}^{\infty} \frac{1}{m}\left(\sum_{\sigma^{m} x=x} \exp g^{m}(x)-e^{m P(g)}\right) .
$$

The above theorem extends a result of Ruelle [16], ([17, pp. 100-101]). 
4. A counter-example to Ruelle's question

Let $\Sigma_{A}=\left\{x \in \prod_{-\infty}^{\infty}\{1, \ldots, k\} \mid A\left(x_{n}, x_{n+1}\right)=1\right\}$ then $\sigma: \Sigma_{A} \rightarrow \Sigma_{A}$ given by $(\sigma x)_{n}=x_{n+1}$ is a (two-sided) shift of finite type. Let $f: \Sigma_{A} \rightarrow \mathbb{R}^{+}$be a strictly positive continuous function for which there exists $0<\theta<1, C>0$ satisfying $|f(x)-f(y)| \leq C \theta^{n}$ whenever $x_{i}=y_{i},|i| \leq n-1$. Define

$$
\Sigma_{A}^{f}=\{(x, t) \mid 0 \leq t \leq f(x)\},
$$

where $(x, f(x))$ and $(\sigma x, 0)$ are identified. The $f$ suspension $\sigma_{t}^{f}: \Sigma_{A}^{f} \rightarrow \Sigma_{A}^{f}$ is the flow defined by $\sigma_{t}^{f}(x, s)=(x, t+s)$ with appropriate identifications. Thus $\sigma^{f}$ can be interpreted as flowing vertically under the graph of $f$. The flow $\sigma^{f}$ is (topologically) weak mixing if the rank of $\Gamma_{f}=\left\langle\exp f^{n}(x) \mid \sigma^{n} x=x\right\rangle$ is greater than one [12]. The topological entropy of $\sigma^{f}$ is the unique $h \in \mathbb{R}^{+}$satisfying $P(-h f)=0$ [12]. The zeta function associated with $\sigma^{f}$ is

$$
Z(s)=\zeta(-s f)=\exp \sum_{m=1}^{\infty} \frac{1}{m} \sum_{\sigma^{m} x=x} \exp -s f^{m}(x) .
$$

In [12] Parry and the author partially answered a question of Ruelle ([17, p. 173]) by showing that $Z(s)$ has an analytic extension to a neighbourhood of $\{s \mid \mathscr{R}(s) \geq h\}$, except for a simple pole at $s=h$. We shall now complete this analysis by presenting a flow for which $Z(s)$ is not analytic on any strip $h-\delta<\mathscr{R}(s)<h$.

Let $\sigma: \Sigma_{A} \rightarrow \Sigma_{A}$ be a full shift on two symbols $\{1,2\}$. Choose $0<p<\frac{1}{2}, p+q=1$, and define a locally constant function $f$ by

If

$$
f(x)= \begin{cases}-\log p & \text { if } x_{0}=1 \\ -\log q & \text { if } x_{0}=2\end{cases}
$$

$$
P^{s}=\left(\begin{array}{ll}
p^{s} & q^{s} \\
p^{s} & q^{s}
\end{array}\right)
$$

where $s \in \mathbb{C}$, then

$$
\sum_{\sigma^{m} x=x} \exp -s f^{m}(x)=\operatorname{trace}\left(P^{s}\right)^{m}=\left(p^{s}+q^{s}\right)^{m} .
$$

Thus

$$
\begin{aligned}
Z(s) & =\exp \sum_{m=1}^{\infty} \frac{1}{m}\left(p^{s}+q^{s}\right)^{m} \\
& =\exp \left(-\log \left(1-p^{s}-q^{s}\right)\right)=1 /\left(1-p^{s}-q^{s}\right)
\end{aligned}
$$

and the poles for $Z(s)$ are the solutions to $p^{s}+q^{s}=1$. In particular the first part of the question shows $h=1$ and for $\sigma^{f}$ to be weak mixing we require $\log p / \log q$ to be irrational.

Let $\varepsilon>0$ satisfy $p^{-\varepsilon}-q^{-\varepsilon}=1$, then the poles are contained in the strip $-\varepsilon \leq \mathscr{R}(s) \leq$ 1. If $-\varepsilon<\sigma<1$, then zero is a limit point of $\left\{p^{s}+q^{s}-1 \mid \mathscr{R}(s)=\sigma\right\}$. Sirce $p^{s}+q^{s}-1$ is an analytic almost periodic function it has a zero in every vertical strip containing $\sigma([2$, p. 75$])$. We conclude that the poles $\left\{\sigma_{n}+i t_{n}\right\}$ for $Z$ are distributed with $\left\{\sigma_{n}\right\}$ dense in the interval $[-\varepsilon, 1]$. (In fact sharper estimates about the distribution of poles are possible (cf. [8])). 
5. A counter-example to Bowen's question

In [5] Gallovotti gave an example of a suspension for which the corresponding zeta function has an essential singularity at $s_{1}<0$. Bowen asked whether the zeta function for flows could always be extended to $s=0([1, \mathrm{p} .31])$. In this section we give an example where this is not the case. In fact it is possible to construct a suspension with an essential singularity at $s_{0}>0$.

Let $\sigma_{n}: \Sigma_{n} \rightarrow \Sigma_{n}$ be a full shift on $n$-symbols and let $\left\{\beta_{k}\right\}$ be a convergent sequence with limit $\beta$. For $n=3$ define $g \in C\left(\Sigma_{3}\right)$ by

$$
g(z)= \begin{cases}\beta_{k} & \text { if } z_{k}=2, z_{i} \in\{1,3\}, 0 \leq i \leq k-1 \\ \beta & \text { if } z_{i} \in\{1,3\}, i \geq 0 .\end{cases}
$$

Let $\sigma^{m} z=z$ and assume the cycle $\left(z_{0}, \ldots, z_{m-1}\right)$ contains disjoint blocks of 1's and 3 's of lengths $k_{1}, \ldots, k_{r}$ with $k_{1}+\cdots+k_{r}=N$. Then

$$
g^{m}(z)=(m-N) \beta_{0}+\sum_{p=1}^{r}\left(\beta_{1}+\cdots+\beta_{k_{p}}\right) .
$$

Thus $g^{m}(z)$ is independent of the $2^{N}$ possible combinations of 1's and 3's.

For $n=2$ define $f \in C\left(\Sigma_{2}\right)$ by

$$
f(x)=\left\{\begin{array}{lc}
\beta_{k}+\log 2 & \text { if } x_{k}=2, x_{i}=1 \\
& 0 \leq i \leq k-1, \quad(k \neq 0) \\
\beta_{0} & \text { if } x_{0}=2 \\
\beta+\log 2 & \text { if } x_{i}=1, i \geq 0 .
\end{array}\right.
$$

The functions $f$ and $g$ are related by

$$
\sum_{\sigma^{\prime \prime} x=x} \exp f^{m}(x)=\sum_{\sigma^{m} z=z} \exp g^{m}(z)
$$

and so $\zeta(f)=\zeta(g)$.

The function $f$ is similar to the Fisher potential used by Gallovotti [5].

Define a locally constant function $f_{N}$ by replacing $\beta_{k}$ by $\beta$ for $k \geq N$. The zeta function $\zeta\left(f_{N}\right)$ can be calculated simply. Define

$$
P_{N}=\left(\begin{array}{ccccc}
e^{\beta_{0}} & 2 e^{\beta_{1}} & \cdots & 2 e^{\beta_{N-1}} & 2 e^{\beta} \\
e^{\beta_{0}} & & & & 0 \\
& 2 e^{\beta_{1}} & & 0 & \vdots \\
& & \ddots & 2 e^{\beta_{N-1}} & 2 e^{\beta}
\end{array}\right)
$$

then by $([14$, p. 82$])$

$$
\begin{aligned}
1 / \zeta\left(f_{N}\right)= & \operatorname{det}\left(I-P_{N}\right) \\
& =\left(1-2 e^{\beta}\right)\left(1-\sum_{n=0}^{N-1} 2^{n} e^{\beta_{0}+\cdots+\beta_{n}}\right)-2^{N} \cdot e^{\beta+\beta_{0}+\cdots+\beta_{N-1}}
\end{aligned}
$$


Assume that $g \in \mathscr{F}_{\theta}$ and $g>0$. By replacing $\beta_{k}$ by $-s \beta_{k}$ (and $\beta$ by $-s \beta$ ) we have from $(5.1)$ and $\S 3$

$$
1 / Z(s)=1 / \zeta(-s g)=\left(1-2 e^{-s \beta}\right)\left(1-\sum_{n=0}^{\infty} 2^{n} e^{-s\left(\beta_{0}+\cdots+\beta_{n}\right)}\right)
$$

(for $\mathscr{R}(s)$ large). In particular, for $2 e^{-\mathscr{R}(s) \beta}<1$ we have $\lim _{N \rightarrow \infty} 2^{N} e^{-s\left(\beta_{0}+\cdots+\beta_{N-1}\right)}=0$. Following Gallovotti we set

$$
\beta_{m}= \begin{cases}-\log \left(\frac{1+\theta^{m} / m}{1+\theta^{m-1} / m-1}\right)+C & m \geq 2 \\ -\log (1+\theta)+C & m=1 \\ C & m=0\end{cases}
$$

and $\beta=C$ (where $C>0$ is chosen to make $\beta_{m}>0$ ). From (5.2)

$$
1 / Z(s)=\left(1-2 e^{-s C}\right)\left(1-\frac{1}{2} \sum_{m=1}^{\infty}\left(1+\theta^{m} / m\right)^{s}\left(2 e^{-s C}\right)^{m+1}-e^{-s C}\right)
$$

Thus the entropy of $\sigma^{g}$ is the solution $h>0$ to

$$
1=\frac{1}{2} \sum_{m=1}^{\infty}\left(1+\theta^{m} / m\right)^{h}\left(2 e^{-h C}\right)^{m+1}+e^{-h C} .
$$

$Z(s)$ has a meromorphic extension to $s=h$ given by

$$
\begin{gathered}
1 / Z(s)=\left(1-2 e^{-s C}\right)\left(1-e^{-s C}[F(s)+1]\right)-2 e^{-2 s C}, \\
F(s)=\sum_{m=1}^{\infty}\left(2 e^{-s C}\right)^{m}\left[\left(1+\theta^{m} / m\right)^{s}-1\right] .
\end{gathered}
$$

For $0<s \leq h$ there exist $B, D>0$ such that

$$
B \cdot s \cdot \theta^{m} / m \leq\left(1+\theta^{m} / m\right)^{s}-1 \leq D \cdot s \cdot \theta^{m} / m
$$

Thus

$$
B \cdot \log \left(1-2 e^{-s C} \theta\right) \leq F(s) / s \leq D \cdot \log \left(1-2 e^{-s C} \theta\right) .
$$

Consider $s_{0}=1 / C \log 2 \theta$. If $s_{0}>0$ (or equivalently $\theta>\frac{1}{2}$ ) then as $s$ approaches $s_{0}$ from above $|F(s)|$ is unbounded but $\left(s-s_{0}\right) F(s)$ tends to zero. If $s_{0}=0$ (or equivalently $\left.\theta=\frac{1}{2}\right)$ then as $s$ approaches zero from above $|s F(s)|$ is unbounded but $s^{2} F(s)$ tends to zero. We conclude that in either case $s_{0}$ is an essential singularity.

Remark. Hofbauer used the Fisher potential to produce examples of functions with two equilibrium states (one a single atom) [7]. The type of functions studied in this section give examples with two non-atomic equilibrium states (one with support a Cantor set).

Remark.' Our example extends in a natural way to suspensions over $\Sigma_{n}, n>3$. This enables us to give an example with an essential singularity $s_{0}$ arbitrarily close to $h\left(\sigma^{\mathrm{g}}\right)=1$.

\section{REFERENCES}

[1] R. Bowen. On Axiom A Diffeomorphisms. Am. Math. Soc. Regional Conf. Proc. No. 35, 1978.

[2] C. Corduneanu. Almost Periodic Functions. Interscience: New York, 1968. 
[3] N. Dunford \& J. T. Schwartz. Linear Operators, Part I. Interscience: New York, 1958.

[4] P. Ferrero \& B. Schmitt. Ruelle's Perron-Frobenius theorem and projective metrics. Colloq. Math. Soc. János Bolyai 27 (1979), 333-336.

[5] G. Gallovotti. Funzioni zeta ed insiemi basilar. Accad. Lincei. Rend. Sc. fismat. e nat. 61 (1976), 309-317.

[6] F. R. Gantmacher. The Theory of Matrices, vol. II. Chelsea: New York, 1974.

[7] F. Hofbauer. Examples of the non-uniqueness of the equilibrium state. Trans. Amer. Math. Soc. 228 (1977), 223-241.

[8] B. Jessen \& H. Tornhave. Mean motions and almost periodic functions. Acta Math. 77 (1945), 137-279.

[9] M. Krasnoselskii. Positive Solutions of Operator Equations. P. Noordhoff: Groningen, 1964.

[10] A. N. Livsic. Cohomology of dynamic systems. Math. USSR Izvestiza 6 (1972), 1276-1301.

[11] W. Parry. Bowen's equidistribution theory and the Dirichlet density theorem. Ergod. Th. \& Dynam. Sys. 4 (1984), 117-134.

[12] W. Parry \& M. Pollicott. An analogue of the prime number theorem for closed orbits of Axiom A flows. Annals of Math. 118 (1983), 573-591.

[13] W. Parry \& K. Schmidt. Natural coefficients and invariants for Markov shifts. Invent. Math. 76 (1984), 1-14.

[14] W. Parry \& S. Tuncel. Classification Problems in Ergodic Theory. London Math. Soc. Lecture Notes 67. Cambridge University Press: Cambridge, 1982.

[15] D. Ruelle. Statistical mechanics of a one-dimensional lattice gas. Commun. Math. Phys. 9 (1968), 267-278.

[16] D. Ruelle. Generalised zeta functions for Axiom A basic sets. Bull. Amer. Math. Soc. 82 (1976), 153-156.

[17] D. Ruelle. Thermodynamic Formalism. Addison-Wesley: Reading, 1978.

[18] D. Ruelle. Flows which do not exponentially mix. C. R. Acad. Sci. Paris 296 Série I, No. 4 (1983), 191-194.

[19] A. E. Taylor. An Introduction to Functional Analysis. Wiley: New York, 1964.

[20] P. Walters. Ruelle's operator theorem and g-measures. Trans. Amer. Math. Soc. 214 (1975), 375-387.

[21] P. Walters. An Introduction to Ergodic Theory. Graduate Texts in Maths. 79. Springer-Verlag: Heidelberg-Berlin-New York, 1981. 